\title{
Hu Shis politische Einstellung und seine Reformideen um die Zeit der 4.-Mai- Bewegung
}

\section{Zur Person von Hu Shi}

Hu Shi wurde am 7. 12. 1891 als Sohn Hu Chuans, eines Beamten der späten Qing-Dynastie, in einem Distrikt der Provinz Jiangsu geboren, der heute zur Stadt Shanghai gehört. 1893 ging er, kaum zwei Jahre alt, mit seiner Mutter zu seinem Vater nach Taiwan, der dort als lokaler Verwaltungsbeamter arbeitete. 1894 kehrte er mit der Mutter zum Herkunftsort der Eltern nach Süd-Anhui zurück. Denn die Situation war nach dem Ausbruch des Sino-Japanischen Seekrieges in jenem Jahr in Taiwan nicht mehr sicher. Ein Jahr später starb sein Vater.

In Anhui besuchte Hu Shi private Schulen, bis er 1904 von der Mutter nach Shanghai geschickt wurde, um dort bis 1910 die Mittelschule zu besuchen. 1910 wurde er durch eine Prüfung als Regierungsstipendiat für ein Studium in den USA ausgewählt. Er ging im selben Jahr in die USA und begann mit dem Studium der Agrarwirtschaft an der Cornell University, wo er fünf Jahre später dann allerdings einen Masterabschluss in Philosophie erlangte (vgl. Hu 1998a, 3-5).

Im Jahr 1915 begann er mit der Promotion bei John Dewey im Fach Philosophie an der Columbia University. Er legte die Doktorprüfung im Jahr 1917 ab und kehrte danach gleich nach China zurück. Den Doktortitel erhielt er allerdings erst zehn Jahre später, da er seine Dissertation erst im Jahr 1927 in Shanghai drucken ließ, der Columbia University dann 100 Exemplare zur Verfügung stellte und somit erst die Bedingung für die Verleihung des Doktortitels erfüllte (vgl. Hu 2003, 507).

Nachdem Hu Shi seine Alma Mater verlassen hatte, wirkte er von 1917 bis 1938 als Professor für Philosophie und Literaturwissenschaft an der Peking Universität. Diese Jahre waren die wissenschaftlich ertragreichsten seines Lebens, publizierte er doch in dieser Zeit zahlreiche Artikel und Aufsätze sowie seine wichtigen Bücher wie Philosophiegeschichte der chinesischen Antike (entstanden im Jahr 1918), Eine komplette Ideengeschichte des chinesischen Mittelalters (entstanden 1920-1930), Eine kleine Ideengeschichte des chinesischen Mittelalters (entstanden im Jahr 1932). ${ }^{1}$

1 Chinesischsprachige Titel und Zitate in diesem Beitrag wurden vom Verfasser selbst ins Deutsche übersetzt.

Ә Open Access. (c) 2021 Qin Mingrui, publiziert von De Gruyter. (cc) BY-NC-ND Dieses Werk ist lizensiert unter einer Creative Commons Namensnennung - Nicht-kommerziell - Keine Bearbeitung 4.0 International Lizenz. 
Es folgten wechselhafte Jahre. Nach einem Intermezzo als Botschafter der Republik China in den USA von 1938 bis 1942 arbeitete Hu Shi zunächst bis 1946 in verschiedenen Institutionen in Amerika, u. a. in der Library of Congress und an der Harvard University, kehrte im Juli 1946 wieder nach China zurück, wo er bis 1948 Rektor der Peking Universität war, und ging dann im April 1949 von Shanghai aus in die USA, um dort Unterstützung für die Beendigung des Bürgerkrieges in China zu erlangen. Allerdings war er dabei erfolglos.

Er blieb in Amerika und arbeitete $u$. a. als Direktor der Bibliothek des Instituts für Ostasienwissenschaften der Princeton University.

1957 wurde $\mathrm{Hu}$ Shi für den Nobelpreis vorgeschlagen. Er hat diesen Preis aber nicht erhalten. Im April 1958 siedelte er von Amerika nach Taiwan um, um dort die Funktion des Präsidenten der Zentralen Akademie zu übernehmen.

Am 24. Februar 1962 starb er in Taiwan an einem Herzinfarkt.

\section{Hintergrund der Neue-Kultur-Bewegung}

Das Leben und Wirken von $\mathrm{Hu}$ Shi erschließt sich vor dem zeitgeschichtlichen Hintergrund, der maßgeblich von den Opiumkriegen geprägt war. ${ }^{2}$ Die Niederlagen stellten auch die Politik des Kaiserhofs und der konfuzianisch gebildeten Beamtenschaft in Frage, hatten doch der Kaiser und seine Gefolgsleute China bis dahin selbstherrlich als die größte Macht und die chinesische Zivilisation als aller Welt überlegen angesehen. Nun aber wurde das Land zweimal von Kriegsschiffen und Kanonen der westlichen Mächte in die Knie gezwungen, so dass der militärisch-technische Rückstand Chinas gegenüber dem Westen nicht länger zu übersehen war. Um ihn zu überwinden, wurde die Verwestlichungsbewegung eingeleitet, ${ }^{3}$ man lernte dabei allerdings nur im technisch-industriellen Bereich vom Westen. Im politisch-kulturellen Bereich beharrte man auf der chinesischen Tradition, in der Meinung, dass „das zivilisatorische wie das militärische System Chinas demjenigen der Westler weit überlegen und nur Chinas

2 Erster Opiumkrieg, auch First Anglo-Chinese War genannt: 1840-1842; Zweiter Opiumkrieg, auch The Arrow War oder Anglo-French Expedition to China genannt: 1856-1860.

3 Diese Bewegung verlief zwischen den 1860er und 1890er Jahren. Ihre Vertreter kamen sowohl aus dem Kreis der Zentralregierung als auch aus dem Kreis der mächtigen lokalen Herrscher. Zum ersteren gehörten u. a. der Ministerpräsident und Verteidigungsminister Yi Xin (1833-1898) und sein Stellvertreter Wen Xiang (1818-1876); zum letzteren gehörten u. a. Zeng Guofan (18111872, Feldherr der Militäreinheit der Provinz Hunan), Zuo Zongtang (1812-1885, General der Militäreinheit und Minister der Regierung der Provinz Hunan), und Li Hongzhang (1823-1901, Feldherr der Militäreinheit der Provinz Anhui und Handelsminister der Zentralregierung). 
Waffen denen des Westens weit unterlegen waren“ und dass „China folglich vom Ausland lernen müsste, scharfe Waffen herzustellen, wenn es sich selbst doch stark machen wollte“ (zitiert nach HCGN, 160).

Doch schon vor dem Sturz der letzten Kaiserdynastie im Jahr 1911 und der Gründung der Republik China im Jahr 1912 wurden Stimmen immer lauter, die sich nun auch gegen das traditionelle politisch-kulturelle System Chinas richteten. Die Neue-Kultur-Bewegung, die mit der Gründung der „Zeitschrift für die Jugend“ (《青年杂志》) durch Chen Duxiu im September 1915 begann und direkt in die 4.-Mai-Bewegung mündete, ${ }^{4}$ nahm dann den Antifeudalismus in ihr Programm auf und setzte sich zum Ziel, Demokratie und Wissenschaft westlicher Ausrichtung in China einzuführen. Die Einführung von Demokratie und Wissenschaft sollte nach Vorstellungen der Vertreter der Neue-Kultur-Bewegung dem Zweck dienen, die rückständige chinesische Tradition durch jene beiden fortschrittlichen Institutionen zu ersetzen.

Und hier nun kommt Hu Shi ins Spiel, der ein Mitstreiter der Neue-Kultur-Bewegung neben Chen Duxiu, Li Dazhao, Lu Xun und anderen war. ${ }^{5}$ Da es diesen Intellektuellen darum ging, die traditionelle chinesische Gesellschaft grundlegend zu erneuern, sei diese Gesellschaft nachfolgend kurz umrissen. Sie enthielt im Wesentlichen folgende Elemente:

1) Kaiserlich-dynastisches Herrschaftssystem mit einem bürokratischen Verwaltungssystem (vgl. HCGN, 160 und 271-292). Seit der Gründung der Qin-Dynastie im Jahr 221 v. Chr. hatte sich ein Herrschaftssystem in China herausgebildet, in dem der Kaiser die höchste Macht im Reich besaß und diese Macht innerhalb der Familie des Kaisers vererbt, das heißt in der Regel an einen Sohn des Kaisers weitergegeben wurde. Der Herrschaftsstab im Kaiserhof bestand aus drei Personengruppen: engen Verwandten des Kaisers, Hochgebildeten und wichtigen Führern der Militäreinheiten. Zwischen Ende des 6. und Beginn des

\footnotetext{
4 Chen Duxiu (1879-1942) kam aus der Provinz Anhui, studierte von 1901 bis 1903 und von 1905 bis 1907 in Japan. Er arbeitete von 1916 bis 1919 als Professor für Geisteswissenschaften und Dekan der Fakultät für Geisteswissenschaften an der Peking Universität. 1921 gründete er die Kommunistische Partei Chinas mit Li Dazhao und Mao Zedong. Er starb im Mai 1942 in Sichuan.

5 Li Dazhao (1889-1927) kam aus der Provinz Hebei, studierte von 1913 bis 1916 in Japan und arbeitete seit 1916 als Professor für Wirtschaftswissenschaft und Direktor der Zentralbibliothek der Peking Universität. 1921 gründete er die Kommunistische Partei Chinas mit Chen Duxiu und Mao Zedong. 1927 wurde er auf Befehl des Warlords Zhang Zuolin festgenommen und hingerichtet. Lu Xun (1881-1936) kam aus der Provinz Zhejiang, studierte von 1902 bis 1909 in Japan und arbeitete von 1920 bis 1926 als Professor für Literatur an der Peking Universität. Er galt als eine der führenden Figuren der 4.-Mai-Bewegung und einer der wichtigsten chinesischen Literaturwissenschaftler und Schriftsteller des 20. Jahrhunderts.
} 
20. Jahrhunderts (1905) fand ein staatliches Prüfungssystem Anwendung, mit dessen Hilfe Beamte rekrutiert wurden. Bei diesen Prüfungen, an denen jeder unabhängig von seiner Herkunft teilnehmen durfte, wurden Inhalte der klassischen chinesischen Philosophie - vor allem aber des Konfuzianismus - geprüft. Je nach erreichtem Grad und Prüfungsnoten konnten die Prüflinge für Ämter und Funktionen in der kaiserlichen Zentralregierung, in den Regierungen auf lokalen Ebenen sowie in staatlichen Verwaltungsstäben ausgewählt werden.

2) Konfuzianismus als staatstragendes ethisches System (vgl. Hou et al. 1980, 131-190 und 360-413; Wang 1984, 67-78). Seit Mitte des 2. Jahrhunderts v. Chr. wurde der Konfuzianismus als staatstragendes ethisches System Chinas gepflegt. Er besteht als ethisches System hauptsächlich aus drei Grundregeln und fünf Grundtugenden. Die drei Grundregeln legen drei Herrschaftsverhältnisse fest: Der Monarch herrscht über seine Untertanen, der Vater über seinen Sohn und der Mann über seine Frau(en). In den fünf Grundtugenden wurden die moralischen Maßstäbe für sämtliche Beziehungen zwischen den Menschen in der traditionellen - man kann auch sagen feudalen - Gesellschaft ausgedrückt: Menschlichkeit, Pflichtgefühl, Anstand, Vernunft und Vertrauen. Der Konfuzianismus machte bis Anfang des 20. Jahrhunderts den Hauptinhalt der chinesischen Bildung aus und hat den Eliten in Politik und Bildung dazu gedient, die Gesellschaftsordnung in China aufrechtzuerhalten.

3) Patriarchalisch-patrimoniales Gesellschaftssystem (vgl. Wang 1984, 47-57 und 112-134). Die traditionelle chinesische Gesellschaft setzt sich aus Familien und Clans zusammen. In der Familie herrscht der Ehemann und Vater, im Clan dasjenige männliche Mitglied, das zur ältesten Generation unter den lebenden Clanmitgliedern gehört und zugleich hinsichtlich Bildung und Reichtum der angesehenste Mann des Clans ist. Das Familieneigentum hauptsächlich aus Land und Haus bestehend - wurde unter den Söhnen als Erben gleich verteilt. Das Claneigentum - hauptsächlich aus Ahnentempel und Land bestehend - blieb Kollektivbesitz des Clans. Der Ahnentempel diente für Zeremonien der Ahnenverehrung, während das Land an die Mitglieder des Clans verpachtet wurde. Die Pachtgebühren wurden für die Veranstaltung der Aktivitäten des Clans, für die Unterstützung armer und hilfsbedürftiger Clanmitglieder, für die Förderung begabter Nachkommen des Clans und Ähnliches verwendet. Frauen hatten im Clan keinen eigenständigen Status. Ihre Stellung hing von den Positionen ihres Mannes und ihrer Söhne ab. In der Regel hatten die Frauen keinen Zugang zur Bildung, und ihre Erziehung diente vor allem dazu, später ihre Ehemänner und ihre Familien versorgen zu können, wobei die durch die grausame Praxis des 
Fußbindens entstandenen kleinen Füße symbolisch dafür stehen, dass Frauen sich nicht in weiten Räumen der Außenwelt, sondern nur im Haus zu bewegen hatten.

4) Autarke Naturalwirtschaft. Die traditionelle chinesische Gesellschaft bestand zu über 90 \% aus Landbevölkerung, die jeweils aus Grundbesitzern, selbständigen Bauern und Pächtern von Ackerland bestand. Das Ackerland wurde für den Anbau von Getreide und Wirtschaftspflanzen (Hanf, Baumwolle usw.) verwendet. Die Agrarproduktion diente hauptsächlich der Deckung des Eigenbedarfs der Landbevölkerung. Von Abgaben der Landbevölkerung lebten die im Staatsdienst Arbeitenden; andere Stadtbewohner lebten vom Handel, Handwerk usw.

\section{Hu Shis politische Einstellung}

\subsection{Kritische Einstellung gegenüber der Tradition und Plädoyer für die Einführung von Wissenschaft und Bildung westlicher Ausrichtung in China}

Die politische Einstellung von $\mathrm{Hu}$ Shi um die Zeit der 4.-Mai-Bewegung drückt sich im Wesentlichen in seinen Stellungnahmen zur traditionellen chinesischen Gesellschaft, zu den Hauptproblemen des damaligen Chinas sowie zu den Zielsetzungen der Neue-Kultur-Bewegung aus, die von den Hauptvertretern dieser Bewegung, vor allem von Chen Duxiu formuliert worden sind. Bereits in der ersten Nummer der „Zeitschrift für die Jugend“, die am 15. September 1915 in Shanghai herauskam, publizierte Chen Duxiu als Gründer dieser Zeitschrift einen Leitartikel, in dem er sechs programmatische Grundprinzipien für die Neue-Kultur-Bewegung formulierte. Diese Prinzipien lauteten: Das gesellschaftliche und politische Leben der Jugend soll demokratisch und nicht sklavenartig, progressiv und nicht konservativ, aktiv und nicht passiv, weltoffen und nicht auf Isolierung des eigenen Landes gerichtet, realitätsbewusst und nicht realitätsfern und schließlich wissenschaftlich und nicht spekulativ sein. In den Diskussionen der folgenden Jahre wurden diese Prinzipien in zwei Zielsetzungen der Neue-KulturBewegung zusammengeführt: nämlich der Einführung von Demokratie und Wissenschaft westlicher Ausrichtung in China. Um diese beiden Systeme in China einzuführen, musste man nach Chen Duxiu die chinesische Tradition abschaffen. Im Jahr 1918 schrieb er in Ausgabe 6.1 dieser Zeitschrift:

Um die Herrin Demokratie zu unterstützen, ist man gezwungenermaßen gegen den Konfuzianismus, gegen die rituellen Gesetze, gegen den Zwang zum Keuschsein, gegen die 
alte Ethik und die alte Politik. Um die Wissenschaft zu unterstützen, ist man notwendigerweise gegen die Quintessenz der chinesischen Kultur und gegen die alte chinesische Literatur.

(Zitiert nach Hu 2013a, 143-144)

Hu Shi stimmte darin mit Chen Duxiu völlig überein. Er bezeichnete wie Chen diese beiden Forderungen ironisch als „Sündenfälle“ der Neue-Kultur-Bewegung, weil sie der chinesischen Tradition widersprechen. Hu selbst behauptete, dass das Wesen der Neue-Kultur-Bewegung vor allem von der neuen Einstellung bestimmt wird, die bei verschiedenen Vertretern dieser Bewegung festzustellen ist. Diese neue Einstellung nannte Hu eine „kritisch-wertende Einstellung“, die folgende Merkmale aufweist:

1) Sie zweifelt daran, ob die institutionalisierten Sitten (z. B. die ethische Maxime „Die Frau hat dem Mann zu gehorchen“) heute noch gültig sind und weiter gelten sollen.

2) Sie hinterfragt, ob die Lehren der Weisen der Antike für die heutige Welt noch Bedeutung haben.

3) Sie zweifelt daran, ob die allgemein anerkannten Verhaltensweisen und -regeln sowie die Überzeugungen aus der Tradition richtig sind und ob sie nicht durch bessere ersetzt werden sollen.

Hu meinte wie Friedrich Nietzsche, dass sich die Menschheit damals in einem Zeitalter des Wertewandels befand. Zu einem solchen Zeitalter gehörte in seinen Augen eben diese kritisch-wertende Einstellung. Als Erscheinungen des Wertewandels im damaligen China führte $\mathrm{Hu}$ auf: Die Bewertung der Frauen wandelt sich - früher wurden Frauen mit kleinen Füßen als schön angesehen, heute werden Fußbindungen als hässlich, ja als unmenschlich und grausam angesehen; die Einstellung zum Opiumrauchen hat sich verändert - noch vor 1910 wurde einem als Gast bei privaten Besuchen Opium angeboten, heute ist Opium jedoch streng verboten. Die Einstellung zu den Reformideen der Gruppe um Kang Youwei ${ }^{6}$ (z. B. die Idee, eine ideale Gesellschaft der großen Harmonie aufzubauen) gilt als veraltet, während sie 20 Jahre zuvor noch als absolut neue Ideen viele Menschen im Lande begeistern konnten.

Wenn nun die kritisch-wertende Einstellung das zentrale Element der Neuen Denkströmung darstellt, so weist sie nach $\mathrm{Hu}$ in der realen Entwicklung zwei Tendenzen auf. Zum einen werden reale Probleme der Gesellschaft, der Politik, der Religion usw. diskutiert. Zum anderen werden neue Ideen und Ge-

6 Kang Youwei (1858-1927), chinesischer Denker und (Reform-)Politiker, war die führende Persönlichkeit bei der politischen Reform von 1898, die mit der Ermordung von sechs führenden Intellektuellen durch die kaiserliche Regierung endete. 
danken aus der Wissenschaft und Literatur des Westens sowie neue Ideologien aus dem Westen rezipiert. Hu nennt die beiden Tendenzen diejenige der Problembehandlungen und diejenige der Einführung westlicher Wissenschaften. Auf der Grundlage von Recherchen und Publikationen in den Zeitungen und Zeitschriften, die vor allem kurz vor 1919 gegründet worden waren, hat er die behandelten Hauptprobleme und die aus dem Westen eingeführten Ideen zusammengeführt. Hauptsächlich folgende Fragen und Probleme sieht $\mathrm{Hu}$ Shi als die wichtigsten Themen der Neue-Kultur-Bewegung an: 1) Die Frage nach der Bedeutung des Konfuzianismus in der traditionellen Gesellschaft und nach der Kaiserzeit, also nach 1911. 2) Die Frage der Literaturreform (neue Bewertung der klassischen chinesischen Werke sowie Plädoyer für das Schreiben in modernem Chinesisch). 3) Die Frage der Herausbildung und Verbreitung einer einheitlichen chinesischen Sprache, d. h. einer Standardsprache, die die Menschen in allen Gebieten des Landes mit verschiedenen Dialekten verstehen. 4) Die Frage der Frauenemanzipation. 5) Die Frage der tradierten Keuschheitsbestimmungen für Frauen. 6) Die Frage der Erziehung zum ritualkonformen Verhalten. 7) Die Frage der Bildungsreform (z. B. Schulpflicht für Mädchen). 8) Fragen über die Sitten und Bräuche von Partnerwahl und Heirat. 9) Die Probleme in der Regelung der Vater-Sohn-Beziehung; gemeint ist wohl vor allem die Regelung von Erbangelegenheiten und der Versorgung der arbeitsunfähigen Eltern. 10) Die Frage nach der Reform der theatralischen Darstellungsweise, womit vermutlich die Ersetzung des klassischen Theaters (z. B. der Pekingoper) durch ein Theater moderner Art gemeint ist (vgl. Hu 2013a, 144).

Was die Einführung und Verbreitung westlicher Ideen und Lehren angeht, so stellte $\mathrm{Hu}$ fest, dass verschiedene neugegründete Zeitungen und Zeitschriften wie z. B. „Die Neue Jugend“, „Die Morgenzeitung“(《晨报》), die „Allgemeine Bürgerzeitung“(《国民公报》), die „Neuen Nachrichten“(《时事新报》) usw. zahlreiche Aufsätze und Artikel publiziert haben, um die Werke, Ideen und Theorien von Ibsen, Marx, Kant, Hegel usw. vorzustellen und zu verbreiten.

In Hus Analyse der Ursachen für das verbreitete Interesse an westlichen Ideen und Theorien drückt sich seine politische Einstellung antitraditionalistischer Prägung aus (vgl. Hu 2013a, 145-146). Er meinte, die chinesische Gesellschaft befinde sich in einer Phase des grundlegenden Wandels, in der die moralischen Institutionen, die jahrtausendelang für die Aufrechterhaltung der chinesischen Gesellschaftsordnung gesorgt hätten, nun vor großen Herausforderungen stünden. Sie seien dabei, in der neuen gesellschaftlichen Situation ihre alte Bedeutung zu verlieren. Mit den moralischen Institutionen meinte er vor allem die Maximen der konfuzianischen Lehre. Solange China eine geschlossene Gesellschaft, d. h. frei von westlichen Einflüssen war, kannte der Konfuzianismus als staatstragende, die Gesellschaftsordnung garantierende 
Lehre kaum politisch-ideologische Herausforderungen, die seine Autorität als Sittengesetz in Frage stellten. Aber spätestens seit den Opiumkriegen wurde der Einfluss westlicher Ideen philosophisch-kultureller Natur in China sichtbar. Er verstärkte sich sehr schnell und erlebte Anfang des 20. Jahrhunderts wohl eine Hochphase. Viele Intellektuelle und einige Politiker plädierten dafür, den Konfuzianismus durch einige zentrale Elemente der modernen westlichen Kultur (z. B. Ideen der Gleichheit, der Freiheit, der demokratischen Rechte) zu ersetzen. Daraufhin versuchten die überzeugten Konfuzianer, die Stellung des Konfuzianismus durch gesetzliche Garantien zu festigen. Aber diese Versuche haben heftige Widerstände auf Seiten der Reformer und Modernisierer hervorgerufen. Gegen 1915/1916 erlebten die Aktivitäten zur Verteidigung des Konfuzianismus den Höhepunkt, gleichzeitig waren die Stimmen gegen den Konfuzianismus auch am lautesten. In dieser Situation haben einige Intellektuelle die Quintessenz des Konfuzianismus sorgfältig analysiert. Die Ergebnisse ihrer Analysen haben viele Menschen von den negativen Wirkungen des Konfuzianismus und von seiner Inkompatibilität mit der gesellschaftlichen Situation der damaligen Zeit überzeugt, so dass sie nicht mehr an die überlieferte Idee glaubten, durch die konfuzianische Lehre eine harmonische Gesellschaft aufbauen zu können. Dieser Wandel der politischen Überzeugungen hat dazu geführt, dass ein Gesetzeserlass der Regierung von Duan Qirui ${ }^{7}$ im Jahr 1918, der den Konfuzianismus zur staatstragenden Ideologie erklärte, kaum Beachtung bei der Bevölkerung fand (vgl. Hu 2013a, 146).

Was das Problem der Reform des Schriftchinesischen angeht, so engagierte sich $\mathrm{Hu}$ für die Ersetzung der klassischen chinesischen Schriftsprache durch das moderne Chinesisch. Sein Argument hing mit der Verbreitung des Rechts auf Bildung und auf Chancengleichheit zusammen. Bildung war, so $\mathrm{Hu}$, in China seit Jahrtausenden nur einer kleinen Elite vorbehalten. Die schwer verständliche Schriftsprache diente dieser Elite und der kaiserlichen Beamtenschaft als Herrschaftsinstrument. Die Massen waren ohne Bildung, konnten diese Sprache nicht verstehen - und brauchten es auch nicht. Seit Beginn der modernen Zeitenwende jedoch war Bildung $\mathrm{zu}$ einem allgemeinen Recht des Bürgers geworden, und immer mehr Menschen bemerkten, dass die klassische Schriftsprache die Verbreitung der Bildung behinderte. Die klassische Schriftsprache unterschied sich weitgehend von der Alltagssprache und wurde nur in Lehrbüchern gebraucht. Aus diesem Grund waren fortschrittliche Intellektuelle wie $\mathrm{Hu}$ Shi und Chen Duxiu der Meinung, dass die klassische Schriftsprache durch eine moderne Schriftsprache zu ersetzen sei, die mit dem Hochchinesischen

7 Duan Qirui (1865-1936) herrschte 1916-1920 als praktischer Regierungschef der Nordchinesischen Regierung und 1924-1926 als Ministerpräsident der Republik China. 
identisch ist (vgl. Hu 2013a, 146). Man sollte sozusagen so schreiben, wie man auf Hochchinesisch spricht.

Hu setzte sich für die Einführung von Wissenschaft und Bildung nach westlichen Maßstäben in China ein. Die Gründe und Motive dafür sind vielfältiger Natur. Aber das grundlegende Motiv war doch, dass man mit den alten chinesischen Wissenschaften unzufrieden war, die im Wesentlichen darauf gerichtet waren, die klassischen Texte des Konfuzianismus und des Daoismus auszulegen und $\mathrm{zu}$ interpretieren. ${ }^{8}$ Hingegen schien die geistige Kultur des Westens, also seine Wissenschaft und Bildung, vielen chinesischen Intellektuellen bei der Erklärung der Natur- und Gesellschaftsphänomene überzeugender zu sein. $\mathrm{Hu}$ plädierte mit einigen anderen Intellektuellen deswegen für die Einführung von Wissenschaft und Bildung westlicher Ausrichtung. Er unterschied ziemlich genau zwischen den Motiven, die verschiedene Gruppierungen von Intellektuellen dazu bewegten, diese Meinung zu vertreten. In seinen Augen erkannte ein Teil der Intellektuellen, dass es China nicht nur an technischen Produkten wie Kanonen, Kriegsschiffen, Telekommunikationsmitteln, Eisenbahn usw. mangelte, sondern auch an neuen Ideen, Gedanken und wissenschaftlichen Lehren. Deswegen waren sie für die Einführung der modernen Wissenschaft und Bildung aus dem Westen. Manche andere Intellektuelle bemühten sich um die Einführung und Verbreitung der westlichen Wissenschaft und Bildung, um dadurch der chinesischen Gesellschaft die Errungenschaften von Aufklärung und Kritik nahezubringen. Auf diese Weise wollten sie mit den neuen wissenschaftlichen Erkenntnissen und Ideen aus dem Westen die chinesische Gesellschaft reformieren oder sogar revolutionieren. Wieder andere Intellektuelle waren laut $\mathrm{Hu}$ unfähig, selbst wissenschaftliche Arbeit zu leisten. Sie versuchten, vom Übersetzen westlicher wissenschaftlicher Werke ins Chinesische zu leben. Sie plädierten für die Einführung westlicher Wissenschaft und Bildung, um solchermaßen ihr Einkommen als Übersetzer zu sichern. Gemeinsam war nach $\mathrm{Hu}$ aber allen diesen Intellektuellen die kritische Einstellung gegenüber der traditionellen chinesischen Bildung. Hu selbst allerdings wird man der erstgenannten Gruppe jener Intellektuellen zuordnen.

8 Kritik an der traditionellen Bildung Chinas hat Li Hongzhang, einer der größten Politiker der späten Qing-Zeit, schon im Jahr 1864 geübt. In einem Brief an den mächtigen Prinzen Yi Xin, mit dem er die Verwestlichungsbewegung geführt hat, beklagte er sich darüber, dass sich die Gelehrten und Beamten in China nur darum bemühten, klassische Texte zu studieren und Kalligraphie zu üben. Das, was sie lernen, sei völlig unnütz für die Bewältigung der Probleme der Gesellschaft, die infolge der Angriffe und Invasionen der westlichen Mächte immer komplizierter und dringender werden. Vgl. Jiang 2018, 59-60. 
Insgesamt sah $\mathrm{Hu}$ die Problemorientierung als den Hauptantrieb für die Neue Denkströmung an. Mit Problemen sind hier praktische gesellschaftliche Fragen wie Ungleichheit der Frauen, Analphabetentum und abergläubische Handlungen gemeint, nicht weniger jedoch auch Fragen ideologischer und makropolitischer Art. Als Beweis für das Desinteresse an Ideologien und Theorien der intellektuellen Welt nannte er die Reaktionen auf die Publikationen von chinesischen Gelehrten über die Mehrwerttheorie von Marx und über die Kritik der reinen Vernunft von Kant. Er stellte nämlich fest, dass diese Publikationen kaum Reaktionen hervorgerufen hatten. Hingegen fanden wissenschaftliche Lehren und Methoden des Westens, die der Lösung von praktischen gesellschaftlichen Problemen in China dienen konnten, große Aufmerksamkeit. Dazu gehörten sicherlich der Empirismus und der Pragmatismus, den $\mathrm{Hu}$ bei John Dewey kennengelernt und in China bekannt gemacht hat.

Wenn nun $\mathrm{Hu}$ zufolge die kritische Einstellung den Geist der Neuen Denkströmung ausmacht, so plädiert er dafür, mit dieser Einstellung auch die gesamte chinesische Tradition kritisch und systematisch neu $\mathrm{zu}$ interpretieren. $\mathrm{Hu}$ war der Ansicht, dass es darum gehe, die Ideen und Gedanken der klassischen chinesischen Philosophie in ihrem jeweiligen Entstehungskontext zu durchdringen und aufgrund dessen zu beurteilen, welche von ihnen für die moderne Zeit wertvoll und welche wertlos oder gar verwerflich seien. Hu war mithin keinesfalls für eine totale Negation der traditionellen chinesischen Kultur bzw. für ihre komplette Ersetzung durch die westliche Kultur. Er definierte das Ziel der Neue-Kultur-Bewegung eher als „Rekonstruktion der Zivilisation“ (Hu 2013a, 150-151). Um dieses Ziel zu erreichen, müsse man gesellschaftliche und individuelle Probleme sukzessive lösen, dürfe aber nicht erwarten, dass man dies durch eine Revolution oder eine totale Erneuerung der Gesellschaft bewerkstelligen könne.

\subsection{Konkrete Probleme lösen und große Ideologien verwerfen}

$\mathrm{Hu}$ Shi stellte fest, dass die chinesische Öffentlichkeit um 1919 vor der großen Gefahr stand, sich nur mit Ideologien zu beschäftigen, anstatt sich um das Gemeinwohl zu kümmern. In der Presse ging es meistens um Diskussionen über die Verehrung des Konfuzius, um die Opferzeremonien für den Himmel, um die militärische Stärke und um den Anarchismus als Ideologie. Hu warf den Teilnehmern an diesen Diskussionen vor, dass sie die eigentliche Aufgabe der Intellektuellen vergessen hätten, nämlich die gesellschaftliche Realität im Land zu beobachten und zu analysieren. Er plädierte dafür, alle Theorien und Ideolo- 
gien dazu zu verwenden, die realen gesellschaftlichen Probleme zu beschreiben und zu analysieren (vgl. Hu 2013a, 117).

Er nannte die Diskussionen über Ideologien „leere Reden“. Über Ideologien zu reden, so Hu, sei zwar eine mühselige Arbeit, doch eine nutzlose Tätigkeit; es gleiche der Bemühung eines Arztes, nur Rezepte auswendig zu lernen, aber die Krankheitserscheinungen nicht genau $\mathrm{zu}$ analysieren und $\mathrm{zu}$ diagnostizieren. Die Ideologien, über die diskutiert wurde, seien alle in den westlichen Ländern entstanden. Nach Hu hätten sie dazu gedient, die aktuellen gesellschaftlichen Probleme des jeweiligen Landes zu erfassen und zu lösen. Da China aber vor ganz anderen Problemen stehe, sei es gefährlich für das Land, mit westlichen ideologisch-politischen Konzepten die chinesische Gesellschaft umzugestalten, um ihre Probleme zu lösen. Der Liberalismus z. B. hat laut Hu als Ideologie viele verwerfliche Handlungen in der Weltgeschichte befördert. Würde man diese Ideologie als politische Grundlage für die Umgestaltung der chinesischen Gesellschaft verwenden, so brächte man das Land nur in Gefahr (vgl. Hu 2013a, 118). Auch der Sozialismus wurde Hu zufolge in China ungenau verstanden und willkürlich interpretiert. Der Sozialismus als Begriff sei nicht im originalen Sinn von Marx verstanden, sondern von Intellektuellen und Politikern für eigene Zwecke umgedeutet und gebraucht worden.

$\mathrm{Hu}$ rief deswegen dazu auf, die leeren Reden $\mathrm{zu}$ beenden und sich stattdessen der Bewältigung konkreter politischer und gesellschaftlicher Probleme zuzuwenden. $\mathrm{Zu}$ diesen Problemen rechnete $\mathrm{Hu}$ die Existenzfrage der Rikschafahrer, die Prostitution, die Frage der Integration Chinas in die Volksgemeinschaft, die Frage der Begrenzung der Amtszeit des Präsidenten sowie das Problem der Korruption und der Frauenemanzipation.

Allerdings war Hu nicht der Meinung, dass man sich überhaupt nicht für Theorien und Ideologien interessieren solle. Vielmehr plädierte er dafür, die Theorien als Grundlage für die Erforschung und Analyse der realen gesellschaftlichen Probleme zu benutzen (vgl. Hu 2013a, 120). Er schrieb dazu:

Meine Schlussfolgerung bleibt: Mehr die Probleme erforschen, weniger von Ideologien reden. Alle Ideologien und Theorien sollen selbstverständlich studiert werden, aber wir sollen sie als eine Art hypothetischer Ansicht studieren, doch nicht als absolute Wahrheiten oder als höchste Dogmen. Alle Ideologien und Theorien sollen als Referenz oder als Materialien für vergleichende Studien benutzt werden.

(Hu 1981, 204)

Das Plädoyer für die Erforschung konkreter Probleme drückt zugleich die politische Einstellung von Hu Shi aus. In seinem Artikel „Mein Abweg“ schrieb er:

Ich rede über Politik, nur um für meinen Pragmatismus zu sprechen . . . Pragmatismus ist auch eine Ideologie . . . Aber der Fokus des Pragmatismus ist auf konkrete Tatsachen und Probleme gerichtet, deswegen erkennt er keine grundlegenden Lösungen an. Er erkennt 
nur sukzessive Fortschritte, nur kluge konzeptionelle Überlegungen sowie eigenständige Versuche bei jedem Schritt als echte Evolution an.

(Hu 2013b, 99)

Hu war schon als Oberschüler vom Evolutionismus begeistert. Während seiner Promotion bei John Dewey an der Columbia University war er dann vom Pragmatismus überzeugt und entwickelte aufgrund dessen seine politische Position für den Pragmatismus. Dabei ging es ihm darum, soziale Probleme wie Prostitution, Analphabetentum, Armut usw. zu lösen, ohne die Gesellschaftsordnung zu verändern, die in China halbkoloniale und halbfeudale Züge trug (vgl. Yang 1987, 48). Diese politische Anschauung von $\mathrm{Hu}$ hat eine Auseinandersetzung mehrerer Intellektueller mit ihm hervorgerufen, die vor allem marxistisch orientiert waren und seine politische Anschauung spöttisch als Reformismus bezeichneten. Der Reformismus von Hu setzte vor allem beim Marxismus an, der sich nach der Oktoberrevolution in China zu verbreiten begann. Hu war der Meinung, dass der Marxismus keine Theorie der objektiven Wahrheit, sondern nur eine unzulänglich begründete Ideologie sei. Daraus folgerte er, dass man in China keine Revolution führen solle, wie sie der Marxismus einforderte (vgl. Yang 1987, 48).

Diese Ansicht wurde von den damals führenden Marxisten wie Li Dazhao, $\mathrm{Qu}$ Qiubai und anderen scharf kritisiert. Bei der Kritik von Li Dazhao an $\mathrm{Hu}$ ging es darum, dass die sozialen Probleme wie Unterdrückung der Frauen, Armut, soziale Ungleichheit usw. nicht gelöst werden können, ohne die Gesellschaftsordnung komplett zu erneuern. Li zufolge können die scheinbar konkreten und isolierten Probleme nur bewältigt werden, wenn die Mehrheit der Bevölkerung sie lösen wolle und bei ihrer Lösung mitwirke. Das erfordere eine Massenbewegung, die von einem gemeinsamen Ideal angetrieben werde. Und dieses Ideal kann seiner Meinung nach nur aus dem Marxismus hervorgehen. Aus diesem Grund setzte er sich für die Verbreitung des Marxismus in China ein und forderte, unter der erkenntnistheoretischen und methodologischen Anleitung der marxistischen Lehre die realen sozialen Probleme in China zu analysieren und zu lösen. Er betont dabei vor allem zwei Gesichtspunkte bei Marx. Zum einen weist er auf die Basis-Überbau-Lehre von Marx hin. Nach Lis Verständnis stellt die Wirtschaftsordnung, die aus Produktivkräften und Eigentumsverhältnissen besteht, die Basis der Gesellschaft dar, worauf andere Konstrukte wie das politische System, das Rechtssystem und das ethische System aufbauen. ${ }^{9}$ Gestaltet man die Wirtschaftsordnung um, so werden mithin

9 Li benutzt hier einfach nur Begriffe wie Wirtschaft, Wirtschaftsorganisation, Wirtschaftsstruktur usw., aber noch nicht den Begriff „Basis“, auch nicht die Begriffe Produktivkräfte und Produktionsverhältnisse. Diese Begriffe werden hier von mir [M. Qin] sinngemäß eingeführt. 
auch diese Konstrukte umgestaltet. Werden die wirtschaftlichen Probleme bewältigt, so können auch die anderen Probleme im politischen System, im Rechtssystem, in Familie und Clan sowie Probleme der Frauenrechte und der Arbeiterrechte gelöst werden. Zum anderen betont er die Klassenlehre von Marx. Dabei postuliert er, dass die Gesellschaftsordnung nur grundlegend umgestaltet werden könne, wenn sich die Arbeiterklasse zu sozialen Bewegungen mobilisieren lasse (vgl. „Die Wochenschau“ 1919). Li kündigte eine grundlegende Lösung der gesellschaftlichen Probleme in China an und erklärte sich bereit, vorbereitende Aktivitäten dafür zu organisieren. Kaum zwei Jahre später gründete er mit Chen Duxiu, Mao Zedong u. a. die Kommunistische Partei Chinas, die in der Tat die Arbeiterschaft und die Bauernschaft zu Revolutionen mobilisieren und organisieren konnte, was zur Gründung der Volksrepublik China geführt hat.

Ein anderer marxistisch gesinnter Intellektueller namens Qu Qiubai übte ebenfalls Kritik an Hus Pragmatismus. Nach ihm findet der Pragmatismus in China Verbreitung, weil die bürgerliche Klasse eine solche Ideologie braucht. Er ist der Meinung, dass philosophische Denkströmungen Produkte der Zeit sind, also immer dann entstehen, wenn das vorhandene Gesellschaftssystem erschüttert ist. Wenn ein altes Gesellschaftssystem von einem neuen ersetzt werde, sei dies als eine Folge des Klassenkampfes zu verstehen. Die geistigen Auseinandersetzungen dabei würden dementsprechend von Klasseninteressen bestimmt. Die Verbreitung des Empirismus und Pragmatismus in China um die Zeit der 4.-Mai-Bewegung war deswegen für Qu kein Zufall, weil diese Zeit nach einer neuen Welt- und Lebensanschauung verlangte. Nach den Opiumkriegen, so seine These, wurde die patriarchalische Gesellschaftsordnung durch den internationalen Kapitalismus erschüttert und die neu entstandene bzw. entstehende Ordnung bürgerlicher Art bedurfte ideologischer Unterstützung. Empirismus und Pragmatismus konnten mit ihren positiven Effekten diesem Bedarf gerade gerecht werden. Qu bezeichnete die Einführung und Verbreitung dieser Lehren deswegen als eine ideelle Revolution der Dritten Klasse, also der bürgerlichen Klasse. Für diese Klasse konnten diese Ideologien als ein revolutionäres Instrument dienen, da sie das Handeln des Bürgertums zu entfesseln vermochten: Während der Konfuzianismus mit seinen ethischen Idealen des Junzi (des edlen Menschen), die die literarische Bildung und den Dienst für das Gemeinwohl betonen, Handlungen zur Verwirklichung des Eigeninteresses verwirft, motivieren der Empirismus und der Pragmatismus vor allem die bürgerliche Klasse zu eigennützigen

Daran zeigt sich, dass Li den Marxismus bis dahin nur über einige wenige Sekundärliteratur grob kannte. 
Handlungen ohne jegliche ideologische Bedenken. Man sollte sich auf die Lösung von konkreten Problemen konzentrieren, pragmatisch handeln und arbeiten, ohne an Ideologien zu denken, so Hu. Und das bedeutet nach Qu für die bürgerliche Klasse, dass man das Eigeninteresse frei verwirklichen kann und soll. $\mathrm{Qu}$ stimmte zwar mit Hu darin überein, dass sich China als ein kulturell zurückgebliebenes Land in einer Situation der internationalen Konkurrenz befand und empirisches und pragmatisches Wissen brauchte, um konkurrenzfähig zu werden. Aber zugleich meinte er, das Land brauche auch wissenschaftliche Theorien wie vor allem die marxistische Gesellschaftstheorie, die es erlaubten, die gesellschaftliche Situation in China grundlegend zu analysieren. Aus dieser Analyse könne nur resultieren, dass die etablierte Gesellschaftsordnung revolutionär umgestaltet werden müsse (vgl. Yang 1987, 50-52).

\section{Hu Shis Reformideen}

\subsection{Vorschläge zur politischen Reform}

Am 13. Mai 1922 publizierte Hu mit 15 anderen in China einflussreichen Intellektuellen, darunter zehn Professoren der Peking Universität, einen Text mit dem Titel „Unsere politischen Meinungen“ (vgl. Hu 2013a, 291-292). Darin kommen die Ideen von $\mathrm{Hu}$ zur politischen Reform in besonders klarer Weise zum Ausdruck.

Wie oben erwähnt, gab es zu dieser Zeit in China verschiedene ideologische Strömungen und dementsprechend politische Überzeugungen und Zielsetzungen bei verschiedenen intellektuellen Gruppierungen. Volksdemokratismus, Gilde-Sozialismus, Anarchismus und andere Denkrichtungen gehörten zu den geläufigsten ideologischen Denkrichtungen. Hu rief ohne Parteinahme für irgendeine Ideologie alle Gruppierungen dazu auf, die Präferenzen für ein bestimmtes politisches System aufzugeben und sich auf ein ganz kleines Ziel der politischen Reform zu einigen, nämlich ein „gutes Regime“ zu schaffen. In Übereinstimmung mit dieser Zielsetzung sollten sich alle Parteien zusammenschließen, um gegen die „bösen Kräfte“ im Lande zu kämpfen (vgl. Hu 2013a, 291).

Was ein gutes Regime ist, darüber hat $\mathrm{Hu}$ eine ziemlich genaue Vorstellung. Er definiert ein solches Regime in positiver wie in negativer Hinsicht. In negativer Hinsicht gehöre es zu einem guten Regime, dass es Instanzen umfasst, die Kader und Beamte kontrollieren und gesetzwidrigen Handlungen vorbeugen bzw. diese bestrafen können. In positiver Hinsicht muss ein gutes Regime zwei Bedingungen erfüllen. Erstens muss es danach streben, dass alle 
seinen Instanzen und Institutionen für das Wohl aller Gesellschaftsmitglieder arbeiten. Zweitens muss es die individuelle Freiheit garantieren und die Persönlichkeitsentwicklung der Gesellschaftsmitglieder fördern. Konkret sprach er drei Forderungen für politische Reformen aus. Erstens muss das Regime aufgrund einer Verfassung regieren, denn nur wenn die Politik auf der Verfassung basiert, kann sie berechenbar sein. Zweitens müssen die Arbeiten der Regierung (einschließlich der Finanz- und Personalangelegenheiten) öffentlich bekanntgegeben und kontrolliert werden, damit keine gesetzwidrigen Handlungen unbestraft bleiben. Drittens müssen die politischen Arbeiten der Regierung auf Planung basieren, weil Hu glaubte, dass die Planlosigkeit einen großen Mangel des chinesischen politischen Systems darstelle und jede Planung besser sei als das blinde Herumtasten in der Politik (vgl. Hu 2013a, 291).

Hu stellte fest, dass China in den ersten Jahren nach der Gründung der Republik, also ungefähr zwischen 1912 und 1917/1918, eine friedliche und prosperierende Zeit erlebt hat, in der die politische und gesellschaftliche Ordnung relativ stabil war, da die „schlechten“ und korrupten Kader und Beamten aus der Kaiserzeit die Politik verlassen hatten und nicht mehr so viel Schaden für die Gesellschaft anrichten konnten wie vorher. ${ }^{10}$ Aber um 1917 wurden die „guten“ Politiker der Politik müde und inaktiv, so dass die „schlechten“ Politiker wie Cao Rulin wieder in die Politik zurückkehren konnten. Hu führte eine Reihe negativer Ereignisse jener Zeit auf die Passivität der gut gesinnten Politiker und Intellektuellen einerseits und das Aktivwerden der schlecht Gesinnten andererseits zurück. Zu diesen Ereignissen zählte er die Teilung des Landes in verschiedene Herrschaftsgebiete der Warlords, den Verlust der Äußeren Mongolei, ${ }^{11}$

10 Als Beispiel nennt er den Fall Cao Rulin. Cao (1877-1966) studierte in Japan und war ein Verfechter der konstitutionellen Monarchie. Er war seit 1904 stellvertretender Außenminister der Qing-Dynastie. Nach dem Sturz der Qing-Dynastie durch die Revolution von 1911 wurde er im August 1913 stellvertretender Außenminister der Regierung von Yuan Shikai (1859-1916), der von Peking aus als Präsident der Republik das Land beherrschte. Seit 1916 war er Verkehrsminister, Außenminister, Finanzminister usw. 1915 verhandelte er als Regierungsvertreter mit den Japanern über die sogenannten 21 Ansprüche der Japaner, nahm im Namen der Regierung mehrmals Kredite von japanischen Banken und gab den Japanern als Gegenleistung dazu die Provinz Shandong als Quasi-Kolonie. Er wurde wegen dieser Entscheidungen als Landesverräter angesehen, und sein Haus wurde während der 4.-Mai-Bewegung in Brand gesetzt. Er sperrte sich danach in seiner Wohnung ein und studierte in aller Ruhe Verfassungslehre. Aber etwas später, als die laut $\mathrm{Hu}$ „schlechten“ Politiker wieder in die Politik zurückkamen, übernahm er wieder verschiedene Regierungsämter.

11 Nach der Revolution von 1911 haben sich verschiedene Provinzen, darunter eben die heutige Äußere Mongolei (Mongolische Volksrepublik) für von der Qing-Dynastie unabhängig erklärt. Mehrere Regierungen der Republik China haben danach versucht, die Äußere Mongolei 
den Verkauf von Shandong, ${ }^{12}$ den Krieg in Südwestchina. ${ }^{13}$ Er rief die gut gesinnte Elite des Landes auf, in der Öffentlichkeit energisch für die Wiedererlangung der Einheit des Landes, für Frieden und politische Reformen einzutreten (vgl. Hu 2013a, 292).

Konkret schlägt er vor:

a. Die Herrscher im Norden und Süden sollen offen und ehrlich über die Einheit Chinas miteinander verhandeln, aber nicht heimlich und hinterlistig die Gegenseite zu schwächen versuchen.

b. Die Friedensverhandlungen sollen unter folgenden Bedingungen geführt werden:

- Das Parlament, das im Jahr 1917 aufgelöst worden war, muss wieder eingeführt werden.

- Das Parlament soll dann eine Verfassung erlassen.

- Die Friedensverhandlung soll einen Abrüstungsplan verabschieden, der innerhalb einer bestimmten Zeitfrist verwirklicht werden muss.

- Alle Inhalte der Friedensverhandlungen sollen öffentlich bekanntgegeben werden.

c. Eine strenge Reform des Beamtenwesens soll durchgeführt werden, die vor allem das Problem der permanten Erhöhung der Kader- und Beamtenzahl zu lösen hat. Konkret sind folgende Reformmaßnahmen zu ergreifen:

- Die Zahl der Kader und Beamten sowohl auf der Ebene der Zentralregierung als auch auf der Provinzebene sind strikt einschränkend festzulegen und auch einzuhalten.

wieder in das Land zu integrieren. Diese Versuche scheiterten nicht zuletzt wegen der militärischen Unterstützung Sowjetrusslands für die Äußere Mongolei. Vgl. HCGN, 502-511.

12 Im Jahr 1918 erhob die japanische Regierung der chinesischen Regierung gegenüber Ansprüche auf verschiedene Rechte (etwa das Recht auf den Bau von Eisenbahnlinien, auf Erschließung von Erzressourcen, auf das Betreiben von Telekommunikationswesen usw.) in China, denen vom Regierungschef Duan Qirui (1865-1936), einem Warlord, zugestimmt wurde. Aufgrund dieser Zustimmung verlangte Japan bei der Verhandlung des Versailler Friedensvertrags die Übernahme der deutschen Kolonie in Shandong. Die Aufnahme dieses Anspruchs in den Versailler Vertrag empörte viele Chinesen, die eine Entkolonialisierung Chinas anstrebten, und führte mit anderen Faktoren zusammen zum Ausbruch der 4.-Mai-Proteste. Vgl. HCGN, 494-495.

13 Im Dezember 1915 krönte sich Yuan Shikai, Präsident der Republik China, zum Kaiser, was viele Menschen, vor allem aber Politiker und Intellektuelle empörte, die die Revolution von 1911 getragen hatten. Mehrere Provinzen erklärten sich daraufhin für unabhängig von seiner Herrschaft. In der Südwestprovinz Yunnan gründeten mehrere Warlords und Politiker unter der Führung von Cai E (1882-1916) die sogenannte Landesverteidigungsarmee, um durch militärische Angriffe die Herrschaft von Yuan Shikai zu stürzen. Yuan befahl seinen Truppen, diese Armee zu vernichten, musste aber eine Reihe von militärischen Niederlagen einstecken und am 22. März 1916 abdanken. Vgl. HCGN, 483-484. 
- Die politischen Berater chinesischer wie ausländischer Herkunft sollen alle entlassen werden, bis auf einige wenige Experten, die das Land wirklich dringend braucht.

- Man soll von den Beamtenrekrutierungssystemen der westlichen Länder lernen und die in öffentlichen Diensten Beschäftigten in zwei Kategorien einteilen, nämlich in die Kategorie der lebenslang Angestellten, also der Beamten, und in diejenige der auf Zeit Beschäftigten. Die Beamten sollen durch strenge Fachprüfungen rekrutiert werden, sonst darf niemand Beamter werden.

d. Das Wahlsystem soll dringend reformiert werden. Erstens soll die direkte Wahl des Staatspräsidenten das herkömmliche Wahlsystem ersetzen, in dem die gewählten Kongressmitglieder den Präsidenten bestimmten. Zweitens sollen in Anlehnung an die Wahlgesetze westlicher Länder strenge Strafmaßnahmen gegen Wahlfälschungen eingeführt werden. Zudem soll die Zahl der Kongressmitglieder und auch die Mitgliederzahl der Parlamente auf der Provinzebene radikal reduziert werden.

e. Was die Reform des Finanzwesens angeht, so soll erstens die Buchhaltung aller Regierungs- und Verwaltungsebenen öffentlich kontrollierbar sein und kontrolliert werden. Zweitens soll der Staat aufgrund der Situation der Steuereinnahmen die öffentlichen Ausgaben festlegen.

Hinzuzufügen wäre hier ein Vorschlag von Hu zur Eindämmung der Korruption, den er einige Jahre später gemacht hat (vgl. Hu 1998b, 26). Hu vertrat die Ansicht, dass die Korruption in China dringend zu bekämpfen sei. Die Trennung von Privatem und Öffentlichem wurde nur selten praktiziert, so dass das Streben nach privaten Vorteilen zum Nachteil der öffentlichen Interessen, also Vetternwirtschaft und Korruption, gang und gäbe war. $\mathrm{Hu}$ war der Meinung, dass der Mensch von Natur aus egoistisch sei und man keine Lösung dieser Probleme durch Entwicklung einer öffentlichen Moral bei den Kadern und Beamten erwarten könne, jedenfalls nicht so schnell. Er sah es deswegen als dringend notwendig an, dass man Gesetze und Regelungen erlässt, durch die die Handlungen der Kader und Beamten kontrolliert werden könnten. Er meinte, eine solche Lösung entspreche auch der traditionellen chinesischen Philosophie, die mit der Parole „Man sollte immer zuerst annehmen, dass der Mensch eher ein gemeiner Mann ist als ein edler Mensch" Ordnungsentwürfe vorziehe, die Kontrolle vor Vertrauen und Moral stellten. 


\subsection{Vorschläge zur Modernisierung der Infrastruktur}

In einem anderen Artikel diskutiert Hu Shi eine Publikation des zeitweiligen Kulturattachés der amerikanischen Botschaft in China Arnold und macht dabei Vorschläge zur Modernisierung von Chinas Infrastruktur (vgl. Hu 1998b, 23-24). Sie wurden freilich erst einige Jahrzehnte später realisiert, nämlich in der Reformphase seit Ende der 1970er Jahre. Arnold behauptete, dass China vor drei zentralen Problemen stehe: Erstens müsse China dringend ein Eisenbahnnetz aufbauen, um die wichtigen Städte und Gebiete miteinander zu verbinden. Zweitens müsse China dringend das Bildungs- und Ausbildungswesen aufbauen bzw. erweitern und modernisieren, um qualifizierte Arbeitskräfte ausbilden und somit die Produktivkräfte erhöhen zu können. Drittens müsse ein Kontrollsystem vor allem für die Arbeit der Kader und Beamten entwickelt werden, damit Korruptionshandlungen bestraft bzw. eingedämmt werden könnten (vgl. Hu 1998b, 23). Nach Arnold war China in der Entwicklung meilenweit von den USA entfernt, eben weil China diese drei Probleme nicht gelöst habe. Um den Rückstand Chinas zu veranschaulichen, verglich Arnold die Landflächen, die Gesamtlänge der Eisenbahnlinien sowie die Gesamtzahl der motorisierten Fahrzeuge der beiden Länder (vgl. Hu 1998b, 23).

Dabei stellte er fest, dass die Landfläche von China zwar größer als diejenige von Amerika war, aber die Länge seiner Eisenbahnlinien gerade 1/36 derjenigen von den USA ausmachte, das Zahlenverhältnis der motorisierten Fahrzeuge in China und in den USA 1/1000 und dasjenige der Straßenlänge $1 / 100 .^{14}$

Angesichts der Rückstände Chinas gegenüber den USA und anderen westlichen Ländern stimmte Hu den drei Reformvorschlägen von Arnold völlig zu. Er führte ausführliche Beweise für diese Rückständigkeit an, um auf die Dringlichkeit der Reform hinzuweisen. Was die Verkehrslage, vor allem die Konstruktion der Eisenbahnlinien angeht, so meinte er, China könnte nur wieder ein einheitliches Land werden, wenn alle seine Gebiete mit der Eisenbahn zugänglich seien. Er nannte verschiedene Beispiele und Phänomene, um zu zeigen, dass China wegen des Mangels an Eisenbahnlinien kein einheitliches modernes Land sein konnte. Im Jahr 1926 kam z. B. ein Vertreter der Handelskammer der Provinz Gansu von Nordwestchina zu einer Tagung nach Peking. Um die Strecke von etwa 1800 Kilometern zurückzulegen, hat er 104 Tage, also 2500 Stunden gebraucht.

14 Heute, also etwa 90 Jahre später, sehen die Verhältnisse ganz anders aus. Auf chinesischen Straßen fuhren 2016 ca. 30 Millionen Fahrzeuge mehr als auf amerikanischen Straßen, und die Länge der elektrifizierten Eisenbahnstrecken in China machte 53mal diejenige in den USA aus. 
Und zum Vergleich: Mit dem Flugzeug von Paris nach Peking brauchte man damals gerade 63 Stunden. Ein anderes Beispiel: Wenn jemand von der südwestlichen Provinz Yunnan nach Shanghai reisen wollte, musste er wegen des Fehlens einer Eisenbahnverbindung in China zuerst nach Vietnam fahren, um von dort aus das Schiff zu nehmen, wobei er ein französisches Visum brauchte, da Vietnam damals Kolonie von Frankreich war. Mit ziemlich großer Aufregung kommentierte Hu diese Zustände: „Die anderen fliegen schon über den Ozean, wir kriechen aber noch auf dem Boden!“ (Hu 1998b, 24).

Zum zweiten wies $\mathrm{Hu}$ auf viele Fakten hin, die besagten, dass China im technischen Bereich dringend Fortschritte machen müsste, um den Vorsprung der westlichen Länder aufzuholen. $\mathrm{Zu}$ diesen Fakten gehörten vor allem Rückstände im Telekommunikationswesen und im Verkehrswesen. Er führte wiederum verschiedene Beispiele an. So konnte man, um eine Nachricht im damaligen China zu senden, nur ein Telegramm schicken, das mindestens 1,4 Silber-Yuan (chinesische Währung von damals) kostete und dennoch nicht garantiert ankam, während man in England für umgerechnet 6 Silber-Yuan ein Radio-Abonnement für ein ganzes Jahr erwerben und damit frei Radio hören konnte. Die Arbeiter der westlichen Länder kamen schon mit motorisierten Fahrzeugen zur Arbeit, und ihre Kinder wurden auch schon im Auto zur Schule gebracht, während hochrangige Kader - also Angehörige der Oberschicht - in China noch mit der Rikscha zur Arbeit fuhren.

Um diese Probleme überhaupt lösen zu können, so Hu Shi, müsste man eine psychische Hemmung überwinden und zugeben können, dass China in jeder Hinsicht rückständig war gegenüber dem Westen: nicht nur hinsichtlich der Technik und der materiellen Entwicklung, sondern auch hinsichtlich der politischen, gesellschaftlichen und moralischen Entwicklung (vgl. Hu 1998b, 27). Sich dieser Erkenntnis anzuschließen stellte damals für viele Chinesen ein Problem dar. So erwiderte ein bekannter Professor der Peking Universität namens Gu Hongming auf die Kritik, die Chinesen seien unzivilisiert, weil sie spuckten, in der Nase bohrten, überall urinierten, sich nicht duschten, die Mädchen zum Fußbinden zwängen usw., viele Europäer würden auch ähnliche Dinge tun und die technische Entwicklung habe den Europäern die Kriegführung erleichtert, was zum grausamen Töten im Ersten Weltkrieg - im damaligen China Europäischer Krieg genannt - geführt hätte. Technik sei deswegen auch nicht nur positiv zu bewerten (vgl. Lin 1998, 17-20). Überhaupt dominierte, so $\mathrm{Hu}$, zu jener Zeit in China eine Atmosphäre der Kritik an der westlichen Zivilisation, wobei diese mit der Parole „Nieder mit dem Imperialismus!“ total abgelehnt wurde. Hu vertrat aber die Meinung, dass die Rückständigkeit Chinas und die großen Probleme im Lande zunächst jedoch nicht auf die Invasionen der westlichen Länder zurückzuführen seien, sondern genuin auf die 
chinesische Kultur selbst, also auf die alte Literatur, auf die alten Gedankensysteme und auf die alte Moral und Religion. Um seine These zu stützen, verweist er auf die japanischen Verhältnisse. Japan, so $\mathrm{Hu}$, habe auch unter Invasionen der westlichen Länder gelitten und habe zwangsläufig auch viele ungleiche Verträge mit ihnen schließen müssen. Aber dennoch sei es durch innenpolitische Reformen und durch Lernen vom Westen so stark geworden, dass seine freie Entwicklung nicht mehr aufzuhalten war.

\section{Schlusswort}

Als $\mathrm{Hu}$ Shi geboren wurde, befand sich China in einer Zeit großer Veränderungen. Einige westliche Mächte hatten das Land schon teilweise kolonialisiert und waren immer noch dabei, ihre Interessen in China durchzusetzen. Die letzte Kaiserdynastie war durch mehrere Kriege mit den westlichen Ländern und durch innerchinesische Aufstände und Revolutionen angeschlagen und versuchte mit allen Mitteln, den Umsturz zu verhindern. Die politische Elite und viele Intellektuelle verteidigten die Dynastie in der Regel zwar nicht, sondern rangen um einen Ausweg aus der krisenhaften Situation Chinas, die von Unterdrückung durch die westlichen Länder sowie durch Spaltung, Armut und kulturelle Rückständigkeit bestimmt wurde. Die Stärkung Chinas war ihr verbindendes Ziel. Über Wege und Mittel zur Erreichung dieses Zieles gab es allerdings verschiedene Ansichten. Sie reichen von der Übernahme der technisch-industriellen Errungenschaften des Westens über politische Reformen (z. B. die Einführung von Elementen des modernen westlichen politischen Systems) bis hin zu Forderungen nach dem revolutionären Sturz der Kaiserdynastie.

Als Kind lebte $\mathrm{Hu}$ auf dem Lande und nahm wohl nur wenig von der Situation Chinas wahr. Aber bereits ab dem 13. Lebensjahr konnte er als Mittelschüler in Shanghai ein Bewusstsein von der prekären Situation des Landes gewinnen und auf dem Wege der Lektüre die westliche Gesellschaft kennenlernen. Als er dann mit 19 Jahren zum Studium nach Amerika ging, wurde er unmittelbar mit der westlichen Gesellschaft sowie der westlichen Kultur und Wissenschaft konfrontiert. Auf Grundlage dieser Erfahrungen entwickelte er seine politische Einstellung und seine Ideen zur Reformierung der chinesischen Gesellschaft, die seine Arbeit und sein Wirken nach der Rückkehr in China dann leiteten.

Politisch strebte er nach Einführung von Wissenschaft und Demokratie westlicher Ausrichtung. Da die Republik China formal zwar schon eine parlamentarische Demokratie etabliert hatte, der allerdings aus verschiedenen Gründen 
(Bürgerkriege, Restauration, Machtkämpfe, Korruption) kaum Erfolg beschieden war, versuchte er darauf hinzuwirken, durch Verhandlungen die Bürgerkriege zu beenden und die Landeseinheit wiederherzustellen, Korruption zu bekämpfen, ein gutes Regime sowie eine schlanke und funktionierende Verwaltung aufzubauen. Indessen wandte er sich strikt gegen jede Revolution, die aufgrund irgendeiner Ideologie zustande gebracht werden sollte.

Innerhalb einer ruhigen und stabilen demokratischen Gesellschaftsordnung, so Hus Plan, sollten dann in allen Gesellschaftsbereichen Reformen verwirklicht werden. Die traditionellen Bildungsinhalte sollten durch moderne wissenschaftliche Inhalte ersetzt werden, eine moderne Infrastruktur sollte aufgebaut, die Frauenemanzipation befördert, Analphabetentum beseitigt und Armut bekämpft werden.

Die Ideen und Publikationen von $\mathrm{Hu}$ Shi hatten großen Einfluss auf die Neue-Kultur-Bewegung und die 4.-Mai-Bewegung. Auch wenn die Geschichte nach der 4.-Mai-Bewegung anders verlief, als $\mathrm{Hu}$ Shi sich das erhofft hatte, wächst sein Einfluss seit ca. 40 Jahren in China deutlich. Vieles, was er und die Intellektuellen seiner Generation erreichen wollten, ist heute Realität geworden. So zählt z. B. die Infrastruktur im heutigen China wohl zu den besten Verkehrssystemen der Welt. Andere Ziele, die Hu Shi und die Protagonisten der 4.-Mai-Bewegung formulierten, wurden (noch) nicht erreicht. Mit Hu Shi könnte man schließen, dass China auf dem zukünftigen Weg seiner Entwicklung die westlichen Gesellschaften und ihre Organisationsformen weiterhin studieren sollte (vgl. Hu 1998b, 3-4).

\section{Literaturverzeichnis}

Hu, Shi. Ausgewählte Werke von Hu Shi [《胡适文集》]. Band 1. Hrsg. Zhu Zheng. Guangzhou 2013a.

Hu, Shi. Aufsatzsammlungen von Hu Shi [《胡适文存》]. Band 2. Beijing 2013b.

Hu, Shi. Hu Shi Gesamtausgabe [《胡适全集》]. Band 26. Hefei 2003.

Hu, Shi. Ausgewählte Werke [《胡适文集》]. Bd. 1. Hrsg. Ouyang Zhesheng. Beijing 1998a.

Hu, Shi. Ausgewählte Werke [《胡适文集》]. Band 4. Hrsg. Ouyang Zhesheng. Beijing 1998b.

$\mathrm{Hu}$, Shi. Eine Autobiographie [《胡适的自传》]. Hrsg. Ge Maochun und Li Xingzhi. Shanghai 1981.

Herausgeberkommission für Chinesische Geschichte der Neuzeit. Chinesische Geschichte der Neuzeit [《中国近代史》]. Beijing 1979 [im Text abgekürzt als HCGN].

Hou, Wailu, Zhao Jibin und Du Guoxiang. Gesamte chinesische Ideengeschichte [《中国思想通史》]. Band 1. Beijing 1980 [1957].

Lin, Yutang. „Maschinen und Geist“. Ausgewählte Werke von Hu Shi. Band 4. Hrsg. Ouyang Zhesheng. Beijing 1998. 17-20. 
Wang, Yanan. Studien zur Bürokratie in China [《中国官僚政治研究》]. Beijing 1984 [1981]. Jiang, Tingfu. Chinesische Geschichte der Neuzeit [《中国近代史》]. Beijing 2018.

Yang, Yabin. Geschichte der chinesischen Soziologie [《中国社会学史》]. Jinan 1987. „Die Wochenschau“ [《每周评论》] 35 (1919). 